\begin{tabular}{|c|l|}
\hline Title & Determination of spin-orbit coefficients in semiconductor quantum wells \\
\hline Author(s) & Faniel, S.; Matsuura, T.; Mineshige, S.; Sekine, Y.; Koga, T. \\
\hline Citation & $\begin{array}{l}\text { Physical Review B, 83(11), 115309 } \\
\text { https://doi.org/10.1103/PhysRevB.83.115309 }\end{array}$ \\
\hline Issue Date & 2011-03_15 \\
\hline Doc URL & http://hdl.handle.net/2115/45039 \\
\hline Rights & @2011American Physical Society \\
\hline Type & article \\
\hline File Information & PRB83_11_115309.pdf \\
\hline
\end{tabular}

Instructions for use 


\title{
Determination of spin-orbit coefficients in semiconductor quantum wells
}

\author{
S. Faniel, ${ }^{1}$ T. Matsuura, ${ }^{2}$ S. Mineshige, ${ }^{1}$ Y. Sekine,${ }^{3}$ and T. Koga ${ }^{1,2}$ \\ ${ }^{1}$ Division of Electronics for Informatics, Graduate School of Information Science and Technology, Hokkaido University, \\ N-14, W-9, Kita-ku, Sapporo, Hokkaido 060-0814, Japan \\ ${ }^{2}$ Creative Research Institution, Research Department, Hokkaido University, Hokkaido 001-0021, Japan \\ ${ }^{3}$ NTT Basic Research Laboratories, NTT Corporation, Atsugi, Kanagawa 243-0198, Japan
}

(Received 12 November 2010; published 7 March 2011)

\begin{abstract}
We report the determination of the intrinsic spin-orbit interaction (SOI) parameters for $\mathrm{In}_{0.53} \mathrm{Ga}_{0.47} \mathrm{As} / \mathrm{In}_{0.52} \mathrm{Al}_{0.48} \mathrm{As}$ quantum wells (QWs) from the analysis of the weak antilocalization effect. We show that the Dresselhaus SOI is mostly negligible in this system and that the intrinsic parameter for the Rashba effect, $a_{\mathrm{SO}} \equiv \alpha /\left\langle E_{z}\right\rangle$, is given to be $a_{\mathrm{SO}} m^{*} / m_{e}=\left(1.46-1.51 \times 10^{-17} N_{S}\left[\mathrm{~m}^{-2}\right]\right) e \AA^{2}$, where $\alpha$ is the Rashba SOI coefficient, $\left\langle E_{z}\right\rangle$ is the expected electric field within the QW, $m^{*} / m_{e}$ is the electron effective mass ratio, and $N_{S}$ is the sheet carrier density. These values for $a_{\mathrm{SO}} m^{*}$ were also confirmed by the observation of beatings in the Shubnikov-de Haas oscillations in our most asymmetric QW sample.
\end{abstract}

DOI: 10.1103/PhysRevB.83.115309

PACS number(s): 73.63.Hs, 71.70.Ej, 73.23.Ad, 85.35.Ds

\section{INTRODUCTION}

As a result of the spin-orbit interaction (SOI), the conduction band of a semiconductor experiences spin splittings even at zero magnetic field, if the system lacks spatial inversion symmetry. In quantum well $(\mathrm{QW})$ structures grown in the [001] direction out of zinc blende crystals, ${ }^{1}$ the spin splittings induced by the bulk inversion asymmetry are the linear and cubic Dresselhaus spin splittings, whose effective Hamiltonians are $H_{D}^{(1)}=\beta_{1} k\left\{\sigma_{x} \cos \theta-\sigma_{y} \sin \theta\right\}$ and $H_{D}^{(3)}=$ $\beta_{3} k^{3}\left\{\sigma_{x} \cos (3 \theta)+\sigma_{y} \sin (3 \theta)\right\}$, respectively, ${ }^{2}$ where $\beta_{1}$ and $\beta_{3}$ are the Dresselhaus SOI coefficients, $k$ the electron wave number, $\sigma_{x}$ and $\sigma_{y}$ the Pauli spin matrices, and $\theta$ the azimuthal angle for the wave vector $\mathbf{k}=\left(k_{x}, k_{y}\right)=(k \cos \theta, k \sin \theta)$. The Rashba spin splitting ${ }^{3}$ arises from the structural inversion asymmetry in artificially grown heterostructures, whose effective Hamiltonian is $H_{R}=\alpha k\left\{\sigma_{x} \sin \theta-\sigma_{y} \cos \theta\right\}, \alpha$ being the Rashba SOI coefficient. $\beta_{1}$ and $\beta_{3}$ are both proportional to a single intrinsic parameter $\gamma$, referred to as the bulk Dresselhaus parameter. ${ }^{4}$ The relations $\beta_{1}=\gamma\left(\left\langle k_{z}^{2}\right\rangle-\frac{k^{2}}{4}\right)$ and $\beta_{3}=\frac{\gamma}{4}$ are obtained from the effective mass approximation, ${ }^{5}$ where $\left\langle k_{z}^{2}\right\rangle$ denotes the expectation value of the operator $\hat{k}_{z}^{2} \equiv-\frac{\partial^{2}}{\partial z^{2}}$ with the wave function along the confinement direction $z$. While it is envisioned that $\alpha$ should be proportional to $\left\langle E_{z}\right\rangle$, where $\left\langle E_{z}\right\rangle$ is the expectation value of the $z$ component of the electric field in the pertinent QW excluding the interfacial contribution from the well-barrier boundary, ${ }^{3,6}$ some debates also exist. ${ }^{7}$ There are multiple mechanisms that would cause Rashba-type spin splittings besides $\left\langle E_{z}\right\rangle$, such as band discontinuities, ${ }^{8,9}$ specific bonding arrangements at the heterointerfaces, ${ }^{10}$ and the lattice-strain effect. ${ }^{11}$ By and large, the relation $\alpha \propto\left\langle E_{z}\right\rangle$ is believed to hold in semiconductor QWs, where the value of the proportionality constant $a_{\mathrm{SO}} \equiv \alpha /\left\langle E_{z}\right\rangle$ should be an intrinsic parameter of the material. The determination of the $\gamma$ and $a_{\text {SO }}$ values and the discovery of simple rules to predict the values of $\beta_{1}, \beta_{3}$, and $\alpha$ will not only play essential roles for the development of future spintronics ${ }^{12-15}$ just in the same way the determination of the effective mass values did for the development of the conventional charge-based electronics,${ }^{16}$ but they will also provide useful checks for bandtheory calculations. Thus, the establishment of experimental schemes to determine these SOI coefficients are of paramount importance.

In this paper, we report on the experimental determination of the intrinsic parameter $a_{\mathrm{SO}}$ in $\mathrm{In}_{0.53} \mathrm{Ga}_{0.47} \mathrm{As} / \mathrm{In}_{0.52} \mathrm{Al}_{0.48} \mathrm{As}$ $\mathrm{QWs}^{17}$ using low temperature magnetoconductance measurements. Thus far, the SOI-related spin splittings have been studied through the beatings in the Shubnikov-de Haas $(\mathrm{SdH})$ oscillations, ${ }^{9,18,19}$ analysis of the weak antilocalization (WAL) dips in magnetoconductance measurements, ${ }^{17,20,21}$ the spin-Galvanic effect, ${ }^{13}$ spin-flip Raman scattering, ${ }^{22}$ electron spin resonance, ${ }^{23}$ and time-resolved Faraday rotation. ${ }^{11,24}$ In these measurements, even if the observed spin splittings were considered to be the Rashba type, they were rarely correlated with $\left\langle E_{z}\right\rangle$ to deduce the values of $a_{\mathrm{SO}}$. Besides that $\left\langle E_{z}\right\rangle$ is not a directly measurable quantity, the difficulties also come from the complicated process of disentangling the values of $\alpha, \beta_{1}$, and $\beta_{3}$ from the measured spin splittings.

In the following section, using the symmetrically doped sample KH1-3 (see Appendix A), we will see that the WAL effect is clearly minimized at some carrier density $N_{S}^{*}$, where $\left\langle E_{z}\right\rangle=0$ is realized (Sec. II A). We then obtain the values $\alpha m^{*}$ from fits to the low-field magnetoconductance data using the Golub model, ${ }^{25}$ where $m^{*}$ is the electron effective mass, neglecting $H_{D}^{(1)}$ and $H_{D}^{(3)}$ over $H_{R}$ (Sec. II B). The parameter values prerequisite in this analysis are only the electron mean free path and the electron density, both of which are obtainable from the Hall and/or $\mathrm{SdH}$ measurements directly, making the purely experimental determination of $\alpha m^{*}$ values possible.

\section{EXPERIMENT}

The heterostructures investigated are lattice-matched, $10 \mathrm{~nm}$-thick $\mathrm{In}_{0.53} \mathrm{Ga}_{0.47} \mathrm{As} / \mathrm{In}_{0.52} \mathrm{Al}_{0.48} \mathrm{As}$ QWs grown by metal organic chemical vapor deposition on (001) InP substrates. ${ }^{26}$ We studied 10 different QWs with various doping profiles (see Appendix A). $\mathrm{In}_{0.53} \mathrm{Ga}_{0.47} \mathrm{As}_{\mathrm{s}} / \mathrm{In}_{0.52} \mathrm{Al}_{0.48} \mathrm{As}$ is a technologically important material system for future spintronics because of its strain-free nature, relatively large spin splitting energies, and stable gate-controllability, where the last factor was made possible owing to recent developments in high- $\kappa$ gate insulators. ${ }^{27}$ 
Standard Hall bar samples were fabricated using either a $20 \mathrm{~nm}$-thick $\mathrm{HfO}_{2}$ layer (atomic layer deposition) or a $100 \mathrm{~nm}$-thick $\mathrm{SiO}_{2}$ layer (evaporation) as gate dielectric. The magnetoconductance measurements were performed at the base temperatures of dilution $(T \sim 30 \mathrm{mK})$ or ${ }^{3} \mathrm{He}$ $(T \sim 300 \mathrm{mK})$ refrigerators with the magnetic field $B$ applied perpendicular to the plane of the QW. The electric characterizations were done using a standard lock-in technique with an electric current of $\sim 100 \mathrm{nA}$ and frequencies between 11 and $183 \mathrm{~Hz}$.

\section{A. Determination of the bulk Dresselhaus parameter $\gamma$}

Shown in Fig. 1 are the measured magnetoconductance traces $\sigma(B)$ for a symmetrically doped sample (KH1-3) at several values of gate-controlled $N_{S}$. The negative magnetoconductances, a characteristic signature of the WAL effect, are clearly seen in all traces in the vanishing $B$. A measure of the SOI strength is provided by the characteristic field for the SOI, $B_{\mathrm{SO}}$, which is given as $B_{\mathrm{SO}} \equiv \alpha^{2} m^{* 2} / e \hbar^{3}$ for the case of the Rashba spin splitting. ${ }^{28}$ Empirically, the values of $B_{\text {SO }}$ are approximated by the magnetic field where $\sigma(B)$ is minimized, denoted as $B_{\min }$ (see vertical marks in Fig. 1). Equating $B_{\text {min }}$ to $B_{\text {SO }}$, we obtain a phenomenological SOI coefficient $\tilde{\alpha} m^{*}=$ $\sqrt{e \hbar^{3} B_{\min }}$. In Fig. 2, we find that such $\tilde{\alpha} m^{*}$ has a V-shaped dip as a function of $N_{S}\left(N_{S}^{*}=1.06 \times 10^{16} \mathrm{~m}^{-2}\right.$, where $\tilde{\alpha} m^{*}$ is minimized). In the presence of both the Dresselhaus and Rashba SOIs, the WAL effect is expected to be weakened if $\alpha= \pm \beta_{1},{ }^{14,15,29}$ suggesting a double dip feature in the $\tilde{\alpha} m^{*}$ vs $N_{S}$ curve. However, in our measurements, we observed the weakening of $\tilde{\alpha} m^{*}$ at only one $N_{S}$.

This single minimum behavior in the $\tilde{\alpha} m^{*}$ vs $N_{S}$ relation suggests either (1) $\beta_{1}$ is so small that the $\alpha= \pm \beta_{1}$ conditions are merged together into a single dip, or (2) $\beta_{1}$ is so large that the second minimum is not visible in the investigated

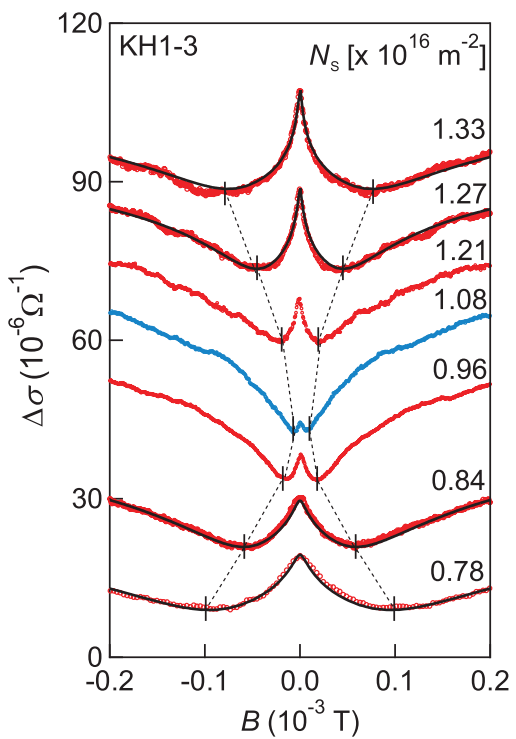

FIG. 1. (Color online) Magnetoconductances of a gated$\mathrm{In}_{0.53} \mathrm{Ga}_{0.47}$ As QW (KH1-3) for various values of $N_{S}$. Each trace has been shifted vertically. The solid curves show the fits to the data by the Golub model. ${ }^{25}$ The position of $B_{\text {min }}$ is indicated by vertical marks in each trace. The dashed lines are guides to the eye.

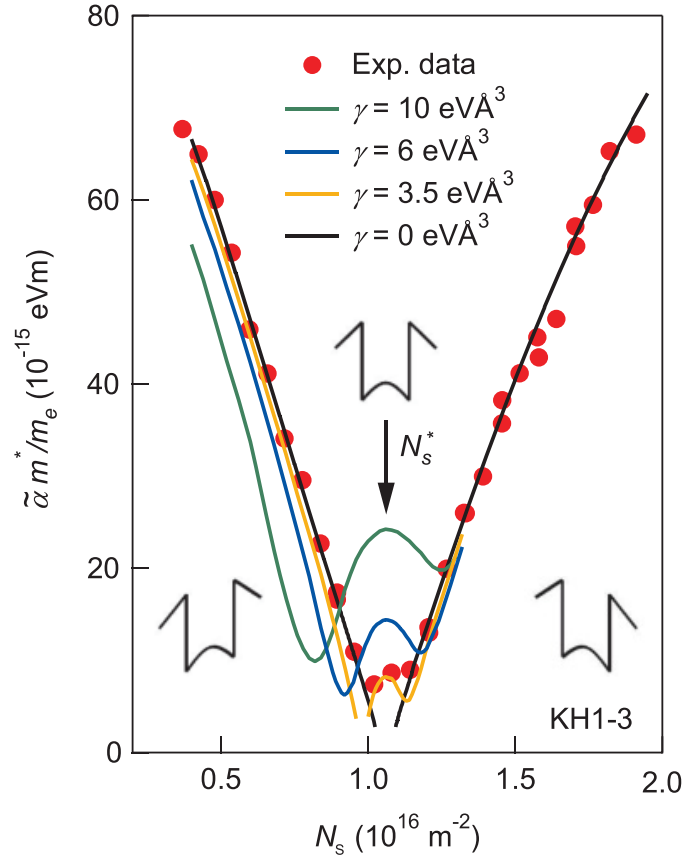

FIG. 2. (Color online) The phenomenological SOI coefficient $\tilde{\alpha} m^{*}$ extracted from the $B_{\min }$ values in Fig 1 . The solid lines are calculations obtained using the $\operatorname{Knap}\left(\gamma=3.5,6\right.$, and $\left.10 \mathrm{eV} \AA^{3}\right)$ and Golub $\left(\gamma=0 \mathrm{eV} \AA^{3}\right)$ models. The vertical arrow indicates the density $N_{S}^{*}$, where the QW is made symmetric; i.e., $\left\langle E_{z}\right\rangle=0$.

$N_{S}$ range. The latter interpretation is ruled out since large $\beta_{1}$ values also imply a strong contribution from $H_{D}^{(3)}$ which is inconsistent with the minimum value of $\tilde{\alpha} m^{*}$ observed at $N_{S}^{*}$ $\left(\tilde{\alpha} m^{*} / m_{e}=7.5 \times 10^{-15} \mathrm{eV} \mathrm{m}\right){ }^{30}$ The minimum in the $\tilde{\alpha} m^{*}$ vs $N_{S}$ curve then corresponds to the condition where the QW is made symmetric; i.e., $\left\langle E_{z}\right\rangle=0$ at $N_{S}^{*}$.

We estimated the value of $\gamma$ as follows. Shown together in Fig. 2 are the simulated values of $\tilde{\alpha} m^{*}$, extracted from the theoretically modeled magnetoconductance curves including the Rashba and Dresselhaus spin splittings and assuming $\gamma=$ $0,3.5,6$, and $10 \mathrm{eV} \AA^{3}$, using the theoretical models developed by $\operatorname{Golub}^{25}\left(\gamma=0 \mathrm{eV} \AA^{3}\right)$ and Knap et al. ${ }^{5}(\gamma=3.5,6$, and $10 \mathrm{eV} \AA^{3}$ ). In these calculations, we used Eq. (4) for the Rashba spin splittings with $N_{S}^{*}=1.06 \times 10^{16} \mathrm{~m}^{-2}$, which will be justified later, and assumed $\tau_{3}=\tau_{\text {tr }}$ when using the Knap model. Here $\tau_{\text {tr }}$ is the transport scattering time and $\tau_{3}$ is a higher order scattering time defined as ${ }^{5}$

$$
\frac{1}{\tau_{3}}=\int_{0}^{\pi} W(\theta)[1-\cos (3 \theta)] d \theta,
$$

where $W(\theta)$ is the probability of scattering by an angle $\theta$. The value of this parameter depends on details of the scattering mechanism-for example, $\tau_{3}^{-1} / \tau_{\text {tr }}^{-1}=1$ for isotropic scattering and $\tau_{3}^{-1} / \tau_{\mathrm{tr}}^{-1}=9$ for small-angle scattering. ${ }^{5}$ In Fig. 2, the condition $B_{\mathrm{SO}} \ll B_{\mathrm{tr}}$, required in the Knap model, is satisfied at $N_{S}$ smaller than $1.3 \times 10^{16} \mathrm{~m}^{-2}$, where $B_{\mathrm{tr}}$ is a characteristic magnetic field relevant to $\tau_{\text {tr }}$, so that the theoretical $\tilde{\alpha} m^{*}$ data for $\gamma=3.5,6$, and $10 \mathrm{eV} \AA^{3}$ are limited to this range. ${ }^{31}$ We found that the simulated $\tilde{\alpha} m^{*}$ vs $N_{S}$ curves substantially deviate from the experimental one if $\gamma>$ 
$3.5 \mathrm{eV} \AA^{3}$. Thus, we regard the value $3.5 \mathrm{eV} \AA^{3}$ as a reasonable upper limit for the experimental $\gamma$. For $\gamma \lesssim 3.5 \mathrm{eV} \AA^{3}$, the double dip of the $\tilde{\alpha} m^{*}$ vs $N_{S}$ curves is restricted to a narrow $N_{S}$ range around $N_{S}^{*}$, where the calculated values of $\tilde{\alpha} m^{*}$ underestimate the experimental ones ${ }^{32}$ (see Appendix B). This discrepancy suggests the presence of additional spin relaxation mechanisms ${ }^{33}$ not included in the theoretical model, or spatial variations of $\alpha$ in the sample, reflecting that of the doping density and/or the QW thickness $d_{\mathrm{QW}}$.

We note that the above estimated value of $\gamma$ is smaller than the predicted values in $\mathbf{k} \cdot \mathbf{p}$ theory $\left(\gamma \sim 27 \mathrm{eV} \AA^{3}\right) .^{5,34}$ A similar observation is found in a recent experiment by Zhou et al. ${ }^{35}$ The $\gamma$ values for GaAs QWs $\left(\gamma \sim 5 \mathrm{eV} \AA^{3}\right)$ are also known to be reduced from the $\mathbf{k} \cdot \mathbf{p}$ value for bulk GaAs $(\gamma \sim$ $\left.27 \mathrm{eV} \AA^{3}\right) .{ }^{15,36}$ After all, we conclude that $\gamma \lesssim 3.5 \mathrm{eV} \AA^{3}$ in (001) $\mathrm{In}_{0.53} \mathrm{Ga}_{0.47} \mathrm{As}(10 \mathrm{~nm}) / \mathrm{In}_{0.52} \mathrm{Al}_{0.48}$ As QWs. Thus, $H_{D}^{(1)}$ and $H_{D}^{(3)}$ may be neglected relative to $H_{R}$ in most cases except at $N_{S}=N_{S}^{*}$ even with sample KH1-3.

\section{B. Determination of the intrinsic parameter for the Rashba effect}

Having shown that $H_{D}^{(1)}$ and $H_{D}^{(3)}$ may be neglected relative to $H_{R}$, we fitted the experimental $\sigma(B)$ data with the WAL theory developed by Golub recently, ${ }^{25}$ assuming only Rashba-type spin splitting, for accurate deduction of the $\alpha m^{*}$ values. In contrast to the previous theoretical models, ${ }^{5,28}$ valid only for $B_{\text {SO }} \ll B_{\text {tr }}$ and $B \ll B_{\text {tr }}$, the new theory is valid for all magnetic field ranges and Rashba coefficient $\alpha$ values. $^{21}$ The excellent quality for the fittings of the WAL data by this model is found in Fig. 1. We also note that it is the value of the product $\alpha m^{*}$, not the value of $\alpha$, that is deducible directly from experiment. Thus, the value of the product $a_{\mathrm{SO}} m^{*}$ is obtained purely experimentally without assuming any values of other parameters, as below.

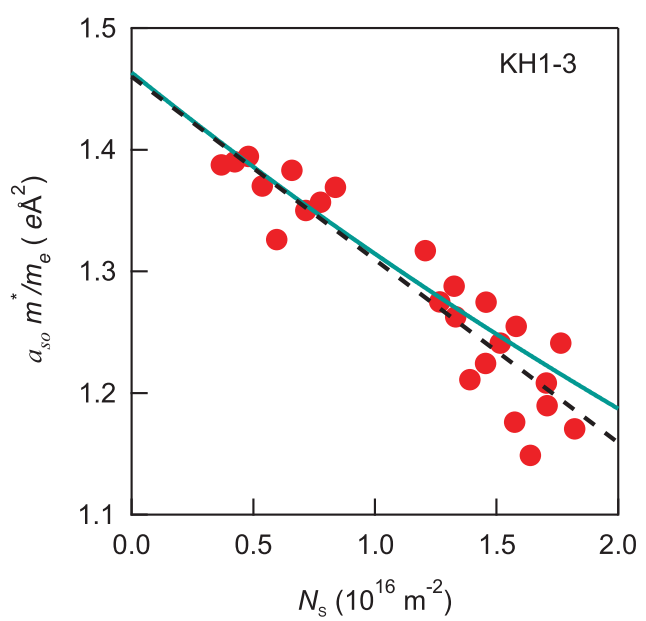

FIG. 3. (Color online) The extracted values of $a_{\mathrm{SO}} m^{*}$ as a function of $N_{S}$ for KH1-3. The dashed line is a linear fit to the data. The solid curve shows the values of $r_{41}^{6 c 6 c} m^{*}$ calculated using Eqs. (2) and (4), replacing $E_{g}$ with $\left(E_{g}+E_{F}\right)$ and multiplied by 1.68 (see text).
Plotted in Fig. 3 are the values of the intrinsic parameter $a_{\mathrm{SO}} m^{*} \equiv \alpha m^{*} /\left\langle E_{z}\right\rangle$ as a function of $N_{S}$, where $\left\langle E_{z}\right\rangle$ is given as $\frac{|e|}{2 \epsilon_{\epsilon} \epsilon_{0}}\left(N_{S}^{*}-N_{S}\right)$ directly from the Poisson equation through the integration by parts ${ }^{34}$ assuming that electrons are entirely confined within the QW, and the dielectric constant used is $\epsilon_{s}=13.1$. We note that the positive and negative values were assigned for $\alpha$ at $N_{S}$ smaller and larger than $N_{\mathrm{S}}^{*}$, respectively, reflecting the sign of $\left\langle E_{z}\right\rangle$. It turned out that the obtained $a_{\mathrm{SO}} m^{*} / m_{e}$ values decrease with increasing $N_{S}$. A linear regression to the experimental data, assuming the formula $a_{\mathrm{SO}} m^{*} / m_{e}=a-b N_{S}$, gave the parameter values $a=1.46 e \AA^{2}$ and $b=1.51 \times 10^{-17} e \AA^{2} \mathrm{~m}^{2}$.

\section{DISCUSSION}

\section{A. Comparison with $\mathbf{k} \cdot \mathbf{p}$ theory}

We now compare the values of $a_{\mathrm{SO}} m^{*}$ obtained in our analysis with predictions from $\mathbf{k} \cdot \mathbf{p}$ theory. In third-order perturbation theory using the extended Kane model, the leading terms of the bulk effective mass $m^{*}$ and $r_{41}^{6 c 6 c}$, the bulk counterpart of $a_{\text {SO }}$, are respectively given as

$$
\frac{m_{e}}{m^{*}}=1+\frac{2 m_{e}}{\hbar^{2}} \frac{P^{2}}{3}\left(\frac{2}{E_{g}}+\frac{1}{E_{g}+\Delta_{\mathrm{SO}}}\right)
$$

and

$$
r_{41}^{6 c 6 c}=\frac{e P^{2}}{3} \frac{2 \Delta_{\mathrm{SO}}\left(E_{g}+\Delta_{\mathrm{SO}} / 2\right)}{E_{g}^{2}\left(E_{g}+\Delta_{\mathrm{SO}}\right)^{2}},
$$

where $E_{g}$ is the band gap energy, $\Delta_{\mathrm{SO}}$ is the spin-orbit split-off energy, and $P$ is the momentum matrix element. ${ }^{34}$ Putting the band parameter values for $\operatorname{In}_{0.53} \mathrm{Ga}_{0.47} \mathrm{As},{ }^{17,34}$ we obtain the value $r_{41}^{6 c 6 c} m^{*} / m_{e}=0.87 e \AA^{2}$, which is about $60 \%$ of the experimental value of $a$. For the observed $N_{S}$ dependence of $a_{\mathrm{SO}} m^{*}$, we note that increasing the Fermi energy $E_{F}$ has a similar effect as increasing the effective band gap energy. ${ }^{34,37}$ Replacing $E_{g}$ in Eqs. (2) and (3) with $\left(E_{g}+E_{F}\right)$, the $N_{S}$ dependence of $r_{41}^{6 c 6 c} m^{*} / m_{e}$ was naively simulated. We then find that the theoretical value of $b$ was also $60 \%$ of the corresponding experimental value (see Fig. 3). The contribution from the valence band discontinuities at the well-barrier interfaces to the SOI can be incorporated into the model by adding the prefactor $\left(\Delta E_{c}+\Delta E_{v}\right) / \Delta E_{c} \approx 1.4$ to Eq. (3), where $\Delta E_{c}$ and $\Delta E_{v}$ are the conduction and valence band offsets. ${ }^{34}$ When including the band discontinuity effect in this way, the theoretical $r_{41}^{6 c 6 c} m^{*}$ is $84 \%$ of the experimental $a_{\mathrm{SO}} m^{*}$. The values of $a_{\mathrm{SO}} m^{*}$ extracted from our experimental data are thus found to be in reasonable agreement with $\mathbf{k} \cdot \mathbf{p}$ theory.

\section{B. Applicability of the obtained $a_{\mathrm{SO}} m^{*}$ values to the other asymmetric quantum wells}

The applicability of the obtained $a_{\mathrm{SO}} m^{*}$ values as a function of $N_{S}$ was checked with the $\alpha m^{*}$ vs $N_{S}$ data obtained for the other $10 \mathrm{~nm}$-thick $\mathrm{In}_{0.53} \mathrm{Ga}_{0.47} \mathrm{As} / \mathrm{In}_{0.52} \mathrm{Al}_{0.48} \mathrm{As}$ QWs that have different back doping densities $N_{1}^{+}$than KH1-3. All these samples showed strong WAL effects in the low temperature magnetoconductance data $\sigma(B)$, where we deduced the $\alpha$ values as a function of $N_{S}$ for each sample from the fittings 

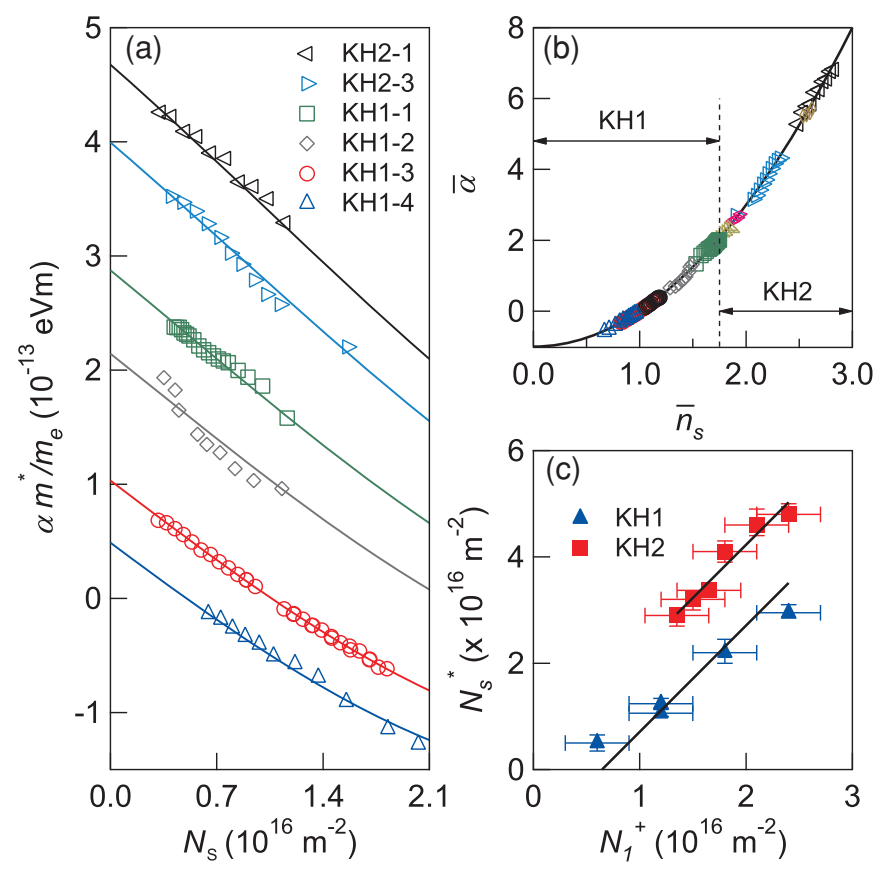

FIG. 4. (Color online) (a) The Rashba parameter $\alpha m^{*}$ as a function of $N_{S}$. The symbols denote the experimental values extracted from the WAL analysis. The solid curves are the fits using Eq. (4). (b) The scaled Rashba parameter values $\bar{\alpha}$ as a function of the scaled electronic density $\bar{n}_{S}$ for all the investigated QWs including those not shown in (a). The relation $\bar{\alpha}=\bar{n}_{S}^{2}-1$ is shown by the solid curve. (c) Virtual electronic densities $N_{S}^{*}$ which correspond to the $\left\langle E_{z}\right\rangle=0$ condition as a function of the doping density $N_{1}^{+}$in the substrate side of the QWs. The solid lines show the expected relation between $N_{S}^{*}$ and $N_{1}^{+}$(line with slope 2). The error bars for $N_{1}^{+}$and $N_{S}^{*}$ represent typical uncertainties by growth and fits to the $\alpha m^{*}$ vs $N_{S}$ curves using Eq. (4), respectively.

with Golub theory as in Fig. 4(a). The experimental $\alpha m^{*}$ vs $N_{S}$ data were then fitted with the equation

$$
\alpha m^{*} / m_{e}=\frac{|e|}{2 \epsilon_{s} \epsilon_{0}}\left(a-b N_{S}\right)\left(N_{S}^{*}-N_{S}\right)
$$

Here, $N_{S}^{*}$ was used as a fitting parameter, which only virtually corresponds to the $\left\langle E_{z}\right\rangle=0$ condition in these QWs. As shown in Fig. 4(a), the experimental $\alpha m^{*}$ vs $N_{S}$ curves are well fitted with Eq. (4) in all samples, if the value of $N_{S}^{*}$ is properly chosen. The generality and applicability of Eq. (4) is summarized in the scaled plot in Fig. 4(b), where the scaled variable $\bar{\alpha} \equiv \alpha m^{*} \frac{8 b \epsilon_{\epsilon_{0}}}{\left(a-b N_{S}^{*}\right)^{2}|e|}$ is plotted as a function of $\bar{n}_{S} \equiv$ $-\frac{2 b N_{S}-a-b N_{S}^{*}}{a-b N_{S}^{*}}$ for all the epiwafers used in the experiment. We find that all the experimental data collapse on a single universal curve $\bar{\alpha}=\bar{n}_{S}^{2}-1$. The validity of our analysis using Eq. (4) is also backed up by the correlation observed between the values of $N_{S}^{*}$ and $N_{1}^{+}$. We note that the change in $N_{1}^{+}$, denoted as $\Delta N_{1}^{+}$, should accompany the change in $N_{S}^{*}$ by $2 \Delta N_{1}^{+}$, if no other background impurities are present below QW and the boundary condition (or the position of the Fermi energy pinning) is kept constant at the buffer layer/substrate interface among the samples, which is roughly the case for a series of samples that were grown under the same growth calibration. This relation is checked for two series of samples investigated

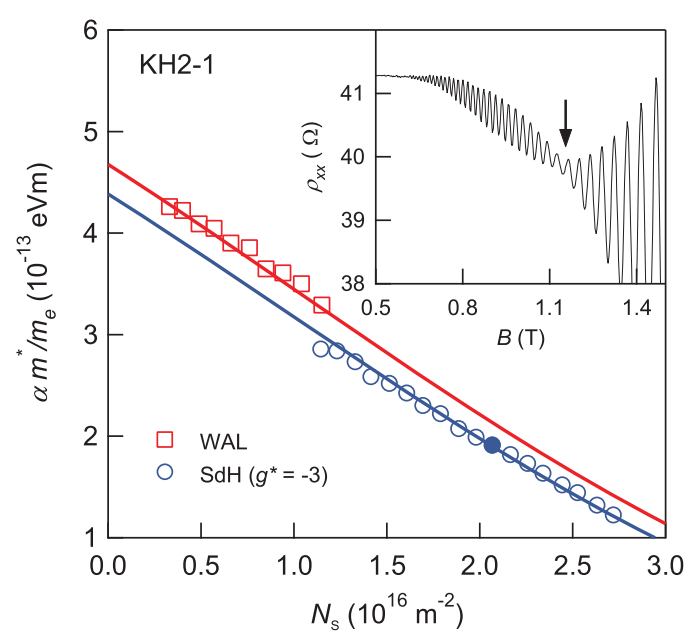

FIG. 5. (Color online) The Rashba parameter $\alpha m^{*}$ as a function of $N_{S}$ extracted for KH2-1 from the WAL (open squares) and $\mathrm{SdH}$ (open circles) analyses. The solid curves are the fits using Eq. (4). Inset: Beating in the $\mathrm{SdH}$ oscillation observed at $N_{S}=2.07 \times$ $10^{16} \mathrm{~m}^{-2}$. The arrow indicates the position of the beating node used for the extraction of the $\alpha m^{*}$ value (filled circle in the main figure). Measurements were performed at $T=30 \mathrm{mK}$ (base temperature).

[Fig. 4(c)], named KH1- $\xi(\xi=1-4)$ and KH2- $\eta(\eta=1-6)$. As shown in Fig. 4(c), good correlations were found between $N_{S}^{*}$ and $N_{1}^{+}$with slope 2 for both $\mathrm{KH} 1$ and $\mathrm{KH} 2$ samples, as expected.

\section{Confirmation by the SdH beating}

We finally report our observation of the $\mathrm{SdH}$ beating with one of our samples that has the largest Rashba spin splitting (KH2-1). An example of such SdH beating patterns is shown in the inset of Fig. 5. In QW samples with well-defined Landau levels, beating nodes appear in the $\mathrm{SdH}$ oscillation at magnetic fields where the spin-splitting energy $\delta\left(\delta \rightarrow 2 \alpha k_{F}\right.$ for $\left.B \rightarrow 0\right)$ is an odd multiple of $\hbar \omega_{c} / 2$, where $\omega_{c}=|e| B / m^{*}{ }^{19}$ Theoretically, the value of $\delta$ is obtained by direct diagonalization of the Hamiltonian matrix using the Landau quantization basis in the presence of the Rashba and Zeeman terms: ${ }^{19}$

$$
\delta=\left\{\left[\hbar \omega_{c}\left(1-g^{*} m^{*} / 2 m_{e}\right)\right]^{2}+\left(2 \alpha k_{F}\right)^{2}\right\}^{\frac{1}{2}}-\hbar \omega_{c},
$$

where $g^{*}$ is the effective Landé $g$ factor and $k_{F}$ is the Fermi wave number. For sample KH2-1, we observed only one node corresponding to $\delta=\hbar \omega_{c} / 2$. Assuming $m^{*} / m_{e}=0.047,{ }^{17}$ and $g^{*}=-3,{ }^{38}$ the extracted values of $\alpha m^{*}$ were plotted as a function of $N_{S}$ in Fig. 5 together with those obtained from the WAL analysis for the same epiwafer (KH2-1). A reasonable agreement is found between the $\alpha m^{*}$ values from the SdH beating and those from the WAL analysis, similar to previous studies in strained InGaAs QWs. ${ }^{21}$ A good fit was also obtained for the $\alpha m^{*}$ vs $N_{S}$ data from the SdH beating using Eq. (4), where the value of $N_{S}^{*}$ turned out to be about $7 \%$ smaller than that from the WAL analysis. This discrepancy may be due to the fluctuations in the back and/or front doping 
densities, namely $N_{1}^{+}$and/or $N_{2}^{+}$, either spatially or by thermal cycling.

\section{CONCLUSION}

As a conclusion, we have deduced the values of the intrinsic parameter $a_{\mathrm{SO}} m^{*} / m_{e}$, which relates the Rashba coefficient $\alpha$ to the averaged electric field within the quantum well $\left\langle E_{z}\right\rangle$, to be $\left(1.46-1.51 \times 10^{-17} N_{S}\left[\mathrm{~m}^{-2}\right]\right) e \AA^{2}$, irrespective of the QW's doping profile, for (001) oriented, $10 \mathrm{~nm}-$ thick gated- $\mathrm{In}_{0.53} \mathrm{Ga}_{0.47} \mathrm{As} / \mathrm{In}_{0.52} \mathrm{Al}_{0.48} \mathrm{As} \mathrm{QWs}$. We have also estimated the value of the bulk Dresselhaus parameter $\gamma$ to be $\lesssim 3.5 \mathrm{eV} \AA^{3}$, implying that the linear and cubic Dresselhaus SOIs are negligible relative to the Rashba SOI in our QWs unless the condition $\left\langle E_{z}\right\rangle \approx 0$ is realized. The quantitative determination of these fundamental parameters is essential for designing semiconductor systems with tailored spin-orbit properties, and provides a useful reference to test band-theory calculations.

\section{ACKNOWLEDGMENTS}

The authors are indebted to Dr. H. Yokoyama and Dr. H. Sugiyama for sample growth, to Professors R. Winkler, L. E. Golub, G. Yu, and J. Nitta for fruitful discussions, and to Dr. K. Sueoka and Dr. T. Akazaki for support. This work was partially supported by KAKENHI, Grant-in-Aid for Young Scientists (A), No. 19684009. S. Faniel thanks the JSPS for financial support.

\section{APPENDIX A}

The heterostructures investigated here are lattice-matched, $10 \mathrm{~nm}$-thick, $\mathrm{In}_{0.53} \mathrm{Ga}_{0.47} \mathrm{As} / \mathrm{In}_{0.52} \mathrm{Al}_{0.48} \mathrm{QWs}$ grown by metal organic chemical vapor deposition (MOCVD) on (001) InP substrates. The growth sequence of the QWs is illustrated in Fig. 6 and all specific growth parameters are summarized Table I.

\section{APPENDIX B}

In Fig. 2, the condition $\tau_{3}^{-1} / \tau_{\mathrm{tr}}^{-1}=1$ may overcount the effect of $H_{D}^{(3)}$ in the simulated magnetoconductance curves

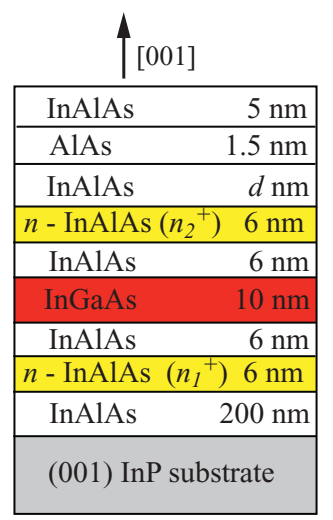

FIG. 6. (Color online) Schematic illustration of the growth sequence for the $\mathrm{In}_{0.53} \mathrm{Ga}_{0.47} \mathrm{As} / \mathrm{In}_{0.52} \mathrm{Al}_{0.48} \mathrm{As} \mathrm{QWs}$.
TABLE I. Growth parameters for the investigated quantum wells: $n_{1}^{+}$and $n_{2}^{+}$are the back and front doping densities by Si dopant, respectively, and $d$ is the thickness of the $\operatorname{In}_{0.52} \mathrm{Al}_{0.48} \mathrm{As}$ Schottky layer. The parameters $n_{1}^{+}$and $n_{2}^{+}$are given in $\left(10^{18} \mathrm{~cm}^{-3}\right)$, and $d$ in $\mathrm{nm}$. Note that $N_{1,2}^{+}=n_{1,2}^{+} \times 6 \mathrm{~nm}$.

\begin{tabular}{cccc}
\hline \hline Wafer & $n_{1}^{+}$ & $n_{2}^{+}$ & $d$ \\
\hline KH1-1 & 4 & 0 & 20 \\
KH1-2 & 3 & 1 & 20 \\
KH1-3 & 2 & 2 & 20 \\
KH1-4 & 1 & 3 & 20 \\
KH2-1 & 4 & 0 & 20 \\
KH2-2 & 3.5 & 0.5 & 20 \\
KH2-3 & 3 & 1 & 25 \\
KH2-4 & 2.75 & 1.25 & 25 \\
KH2-5 & 2.5 & 1.5 & 25 \\
KH2-6 & 2.25 & 1.75 & 25 \\
\hline \hline
\end{tabular}

through the parameter value of $\Omega_{3}^{2} \tau_{3}\left(\Omega_{3}=\beta_{3} k^{3}\right)$ in the Knap model, ${ }^{5}$ because the comparison of the quantum scattering time $\tau_{q}$ and the transport scattering time $\tau_{\text {tr }}$ suggests that
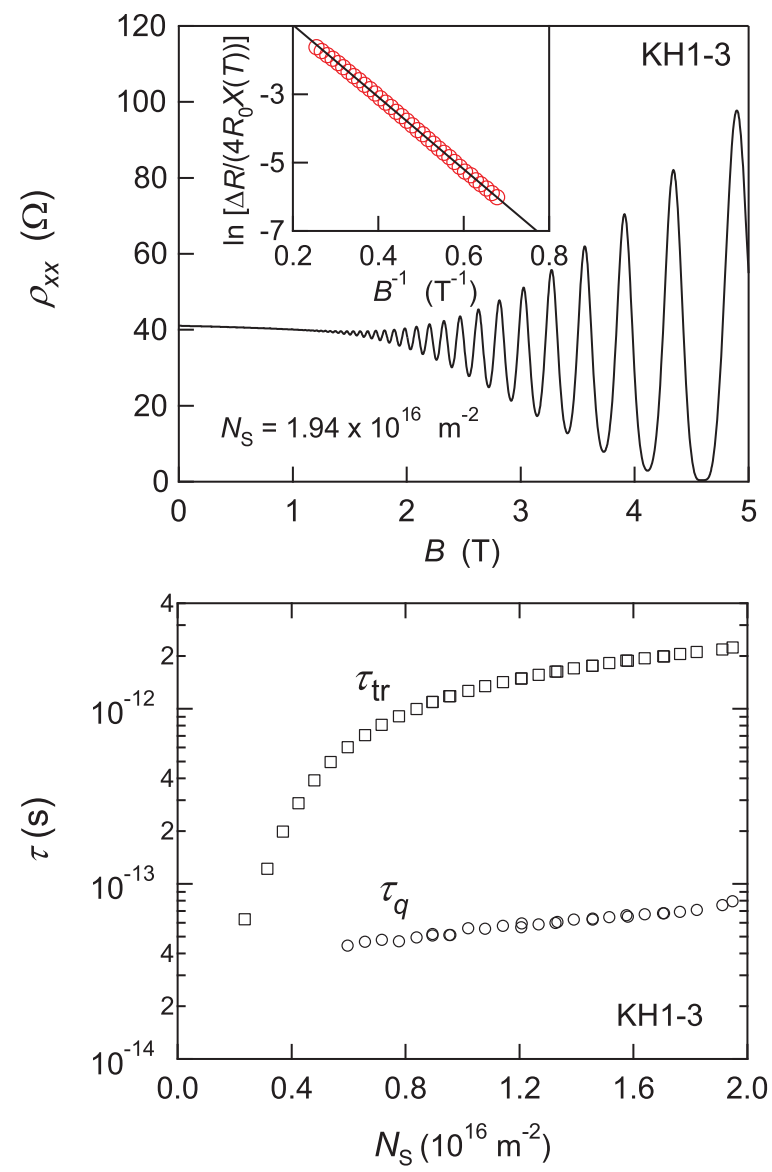

FIG. 7. (Color online) Top panel: Magnetoconductance of KH1-3 at $N_{S}=1.94 \times 10^{16} \mathrm{~m}^{-2}$, showing the $\mathrm{SdH}$ oscillation. Inset: Dingle plot of the $\mathrm{SdH}$ data. The red circles are the experimental data and the plain line is a linear fit to extract the value of $\tau_{q}$. Bottom panel: Transport scattering time $\tau_{\text {tr }}$ and the quantum scattering time $\tau_{q}$ as a function of $N_{S}$ for sample KH1-3. Measurements were performed at $T=30 \mathrm{mK}$ (base temperature). 
forward scatterings dominate in our samples $\left(\tau_{3}^{-1} / \tau_{\mathrm{tr}}^{-1}>1\right)$. The transport scattering time $\tau_{\text {tr }}$ is mostly sensitive to large-angle scattering and rather insensitive to small-angle scattering, while the quantum scattering time $\tau_{q}$ depends on all scattering events. The quantum scattering time $\tau_{q}$ is defined $\operatorname{as}^{39}$

$$
\frac{1}{\tau_{q}}=\int_{0}^{\pi} W(\theta) d \theta,
$$

and also provides a measure of the broadening of the Landau levels. This parameter can be extracted from Dingle plots of the Shubnikov-de Haas data as in Ref. 39. The amplitude of the $\mathrm{SdH}$ oscillation $\Delta R$ is given by

$$
\Delta R=4 R_{0} X(T) \exp \left(\frac{-\pi}{\omega_{c} \tau_{q}}\right)
$$

where

$$
X(T)=\frac{2 \pi^{2} k_{B} T / \hbar \omega_{c}}{\sinh \left(2 \pi^{2} k_{B} T / \hbar \omega_{c}\right)} .
$$

Here, $R_{0}$ is the resistivity at $B=0, k_{B}$ is the Boltzmann constant, $T$ is the temperature and $\omega_{c}$ is the cyclotron frequency. An example Dingle plot is shown in Fig. 7 for $\mathrm{KH} 1-3$ at $N_{S}=1.94 \times 10^{16} \mathrm{~m}^{-2}$. The transport scattering time $\tau_{\text {tr }}$ and the quantum scattering time $\tau_{q}$ are displayed in Fig. 7 as a function of $N_{S}$, for the KH1-3 sample. The quantum scattering time $\tau_{q}$ is found to be typically $1 / 20$ of $\tau_{\text {tr }}$, indicating that forward scatterings dominate in our quantum wells. The assumption $\tau_{3}^{-1} / \tau_{\text {tr }}^{-1}=9$ in the Knap calculation, consistent with forward scattering, reduces the $H_{D}^{(3)}$ contribution to the WAL effect further, leading to a further underestimation, in turn, of the experimental $\tilde{\alpha} m^{*}$ in the vicinity of $N_{S}^{*} \cdot{ }^{32}$
${ }^{1}$ Our crystallographic indices are defined placing the group III and V atoms in the [000] and $\frac{a}{4}[111]$ positions in the unit cell, respectively, where $a$ is the lattice constant.

${ }^{2} \hat{x}\|[100], \hat{y}\|[010]$, and $\hat{z} \|[001]$. $\hat{z}$ is normal to the sample surface.

${ }^{3}$ E. I. Rashba, Fiz. Tverd. Tela 2, 1224 (1960) [Sov. Phys. Solid State 2, 1109 (1960)]; Y. A. Bychkov and E. I. Rashba, J. Phys. C 17, 6039 (1984).

${ }^{4}$ G. Dresselhaus, Phys. Rev. 2, 580 (1955).

${ }^{5}$ W. Knap et al., Phys. Rev. B 53, 3912 (1996).

${ }^{6}$ F. J. Ohkawa and Y. Uemura, J. Phys. Soc. Jpn. 37, 1325 (1974).

${ }^{7}$ A. Därr, J. P. Kotthaus, and T. Ando, in Proceedings of the 13th International Conference on the Physics of Semiconductors, edited by F. G. Fumi (North-Holland, Amsterdam, 1976), p. 774; R. Lassnig, Phys. Rev. B 31, 8076 (1985); S. Brosig, K. Ensslin, R. J. Warburton, C. Nguyen, B. Brar, M. Thomas, and H. Kroemer, ibid. 60, R13989 (1999); A. C. H. Rowe, J. Nehls, R. A. Stradling, and R. S. Ferguson, ibid. 63, 201307 (2001).

${ }^{8}$ P. Pfeffer and W. Zawadzki, Phys. Rev. B 52, R14332 (1995).

${ }^{9}$ G. Engels, J. Lange, T. Schäpers, and H. Lüth, Phys. Rev. B 55, R1958 (1997).

${ }^{10}$ B. Jenichen, S. A. Stepanov, B. Brar, and H. Kroemer, J. Appl. Phys. 79, 120 (1996).

${ }^{11}$ Y. K. Kato, R. C. Myers, A. C. Gossard, and D. D. Awschalom, Science 306, 1910 (2004).

${ }^{12}$ S. Datta and B. Das, Appl. Phys. Lett. 56, 665 (1990); H. C. Koo, J. H. Kwon, J. Eom, J. Chang, S. H. Han, and M. Johnson, Science 325, 1515 (2009); K. C. Nowack, F. H. L. Koppens, Yu. V. Nazarov, and L. M. K. Vandersypen, ibid. 318, 1430 (2007); T. Koga, J. Nitta, H. Takayanagi, and S. Datta, Phys. Rev. Lett. 88, 126601 (2002); S. M. Frolov, S. Lüscher, W. Yu, Y. Ren, J. A. Folk, and W. Wegscheider, Nature (London) 458, 868 (2009); Y. Kato, R. C. Myers, D. C. Driscoll, A. C. Gossard, J. Levy, and D. D. Awschalom, Science 299, 1201 (2003).

${ }^{13}$ S. D. Ganichev, E. L. Ivchenko, V. V. Bel'kov, S. A. Tarasenko, M. Sollinger, D. Weiss, W. Wegscheider, and W. Prettl, Nature (London) 417, 153 (2002).
${ }^{14}$ B. A. Bernevig, T. L. Hughes, and S.-C. Zhang, Science 314, 1757 (2006).

${ }^{15}$ J. D. Koralek, C. P. Weber, J. Orenstein, B. A. Bernevig, S.-C. Zhang, S. Mack, and D. D. Awschalom, Nature (London) 458, 610 (2009).

${ }^{16}$ L. Esaki, Phys. Scr. T 42, 102 (1992).

${ }^{17}$ T. Koga, J. Nitta, T. Akazaki, and H. Takayanagi, Phys. Rev. Lett. 89, 046801 (2002).

${ }^{18}$ B. Das, D. C. Miller, S. Datta, R. Reifenberger, W. P. Hong, P. K. Bhattacharya, J. Singh, and M. Jaffe, Phys. Rev. B 39, 1411 (1989); J. Luo, H. Munekata, F. F. Fang, and P. J. Stiles, ibid. 41, 7685 (1990); J. Nitta, T. Akazaki, H. Takayanagi, and T. Enoki, Phys. Rev. Lett. 78, 1335 (1997).

${ }^{19}$ B. Das, S. Datta, and R. Reifenberger, Phys. Rev. B 41, 8278 (1990).

${ }^{20}$ P. D. Dresselhaus, C. M. A. Papavassiliou, R. G. Wheeler, and R. N. Sacks, Phys. Rev. Lett. 68, 106 (1992); G. L. Chen, J. Han, T. T. Huang, S. Datta, and D. B. Janes, Phys. Rev. B 47, 4084 (1993).

${ }^{21}$ V. A. Guzenko, T. Schäpers, and H. Hardtdegen, Phys. Rev. B 76, 165301 (2007)

${ }^{22}$ B. Jusserand, D. Richards, H. Peric, and B. Etienne, Phys. Rev. Lett. 69, 848 (1992); D. Richards and B. Jusserand, Phys. Rev. B 59, 2506(R) (1999).

${ }^{23}$ M. Schulte, J. G. S. Lok, G. Denninger, and W. Dietsche, Phys. Rev. Lett. 94, 137601 (2005).

${ }^{24}$ L. Meier, G. Salis, I. Shorubalko, E. Gini, S. Schön, and K. Ensslin, Nature Phys. 3, 650 (2007).

${ }^{25}$ L. E. Golub, Phys. Rev. B 71, 235310 (2005).

${ }^{26}$ H. Sugiyama, K. Watanabe, H. Yokoyama, and T. Kobayashi, J. Appl. Phys. 93, 4260 (2003); H. Sugiyama, H. Yokoyama, and K. Wada, Jpn. J. Appl. Phys. 38, 1158 (1999).

${ }^{27}$ I. J. Gelfand, S. Amasha, D. M. Zumbühl, M. A. Kastner, C. Kadow, and A. C. Gossard, Appl. Phys. Lett. 88, 252105 (2006); J. Sun, M. Larsson, I. Maximov, and H. Q. Xu, ibid. 96, 162107 (2010).

${ }^{28}$ S. V. Iordanskii, Yu. B. Lyanda-Geller, and G. E. Pikus, JETP Lett. 60, 206 (1994)

${ }^{29}$ J. Schliemann and D. Loss, Phys. Rev. B 68, 165311 (2003). 
${ }^{30}$ We obtain $\gamma=6 \mathrm{eV} \AA^{3}$ if we attribute this solely to $H_{D}^{(3)}$, assuming $\tau_{3}=\tau_{\text {tr }}$.

${ }^{31}$ The exact criterion used here is $B_{\mathrm{SO}}<\frac{1}{4} B_{\mathrm{tr}}$.

${ }^{32}$ S. Faniel, T. Matsuura, S. Mineshige, Y. Sekine, and T. Koga, in Proceedings of the 30th International Conference on the Physics of Semiconductors (to be published).

${ }^{33}$ G. E. Pikus and A. N. Titkov, in Optical Orientation (Elsevier, Amsterdam, 1984).

${ }^{34} \mathrm{R}$. Winkler, in Spin-Orbit Coupling Effects in Two-Dimensional Electron and Hole Systems (Springer-Verlag, Berlin, Heidelberg, 2003).
${ }^{35}$ Y. M. Zhou, G. Yu, L. M. Wei, K. H. Gao, W. Z. Zhou, T. Lin, L. Y. Shang, S. L. Guo, J. H. Chu, N. Dai, and D. G. Austing, J. Appl. Phys. 107, 053708 (2010).

${ }^{36}$ J. J. Krich and B. I. Halperin, Phys. Rev. Lett. 98, 226802 (2007); see also EPAPS Document No. E-PRLTAO-98-029722.

${ }^{37}$ T. Schäpers, G. Engels, J. Lange, T. Klocke, M. Hollfelder, and H. Lüth, J. Appl. Phys. 83, 4324 (1998); W. Yang and Kai Chang, Phys. Rev. B 74, 193314 (2006).

${ }^{38}$ F. E. Meijer, A. F. Morpurgo, T. M. Klapwijk, T. Koga, and J. Nitta, Phys. Rev. B 70, 201307(R) (2004).

${ }^{39}$ P. T. Coleridge, Phys. Rev. B 44, 3793 (1991). 